\title{
Developmental changes in the ECG of a hamster model of muscular dystrophy and heart failure
}

\author{
Thomas G. Hampton ${ }^{1}{ }^{*}$, Ajit Kale $^{2}$, Scott McCue ${ }^{2}$, Hemmi N. Bhagavan $^{3}$ and Case VanDongen ${ }^{3}$ \\ ${ }^{1}$ The Neuroscience Discovery Core, Framingham, MA, USA \\ ${ }^{2}$ The CuraVita Corporation, Mouse Specifics, Inc., Boston, MA, USA \\ ${ }^{3}$ Research \& Development, BioBreeders, Inc., Watertown, MA, USA
}

Edited by:

Jufeng Wang, Waylandgreen, USA

Reviewed by:

Suyash Prasad, BioMarin

Pharmaceutical, USA

Domenico Criscuolo, Genovax, Italy

*Correspondence:

Thomas G. Hampton, The Neuroscience Discovery Core, Worcester Road, Framingham, MA 01702, USA.

e-mail: hampton@curavita.org

\begin{abstract}
Aberrant autonomic signaling is being increasingly recognized as an important symptom in neuromuscular disorders. The $\delta$-sarcoglycan-deficient BIO TO-2 hamster is recognized as a good model for studying mechanistic pathways and sequelae in muscular dystrophy and heart failure, including autonomic nervous system (ANS) dysfunction. Recent studies using the TO-2 hamster model have provided promising preclinical results demonstrating the efficacy of gene therapy to treat skeletal muscle weakness and heart failure. Methods to accelerate preclinical testing of gene therapy and new drugs for neuromuscular diseases are urgently needed. The purpose of this investigation was to demonstrate a rapid non-invasive screen for characterizing the ANS imbalance in dystrophicTO-2 hamsters. Electrocardiograms were recorded non-invasively in conscious $\sim 9$-month old TO-2 hamsters $(n=10)$ and non-myopathic F1B control hamsters $(n=10)$. Heart rate was higher in TO-2 hamsters than controls $(453 \pm 12 \mathrm{bpm}$ vs. $311 \pm 25 \mathrm{bpm}, P<0.01)$. Time domain heart rate variability, an index of parasympathetic tone, was lower in TO-2 hamsters $(12.2 \pm 3.7 \mathrm{bpm}$ vs. $38.2 \pm 6.8, P<0.05)$, as was the coefficient of variance of the RR interval $(2.8 \pm 0.9 \%$ vs. $16.2 \pm 3.4 \%, P<0.05)$ compared to control hamsters. Power spectral analysis demonstrated reduced high frequency and low frequency contributions, indicating autonomic imbalance with increased sympathetic tone and decreased parasympathetic tone in dystrophic TO-2 hamsters. Similar observations in newborn hamsters indicate autonomic nervous dysfunction may occur quite early in life in neuromuscular diseases. Our findings of autonomic abnormalities in newborn hamsters with a mutation in the $\delta$-sarcoglycan gene suggest approaches to correct modulation of the heart rate as prevention or therapy for muscular dystrophies.
\end{abstract}

Keywords: muscular dystrophy, autonomic nervous system, heart failure, development, cardiomyopathy, hamsters, $\delta$-sarcoglycan deficiency, BIOTO-2 hamsters

\section{INTRODUCTION}

Neuromuscular diseases, routinely considered with regard to muscle weakness, frequently affect the heart and the cardiovascular system. Aberrant autonomic signaling is being increasingly recognized as an important symptom in a variety of clinically defined neuromuscular disorders (Rakocević-Stojanović et al., 2007; Politano et al., 2008; Inoue et al., 2009; Della Marca et al., 2010). Patients with Duchenne muscular dystrophy, for example, often present with tachycardia and significant decreases in heart rate variability (HRV; Marcello et al., 1995; Yotsukura et al., 1998); cardiac failure is frequently the cause of death (Judge et al., 2011). There is a growing number of case reports presenting cardiac abnormalities in patients with spinal muscular atrophy (SMA; Hachiya et al., 2005; Rudnik-Schöneborn et al., 2008), a debilitating neurological disease marked predominantly by motor neuron death and atrophy of skeletal muscle. Cardiac muscle pathology sometimes exceeds skeletal muscle pathology in cases of limbgirdle muscular dystrophy (LGMD; Margeta et al., 2009). Many neuromuscular diseases affect children. Duchenne's muscular dystrophy and some LGMD, childhood muscle disorders resulting from loss of expression of sarcoglycans, are both characterized by similar aberrant autonomic modulation of the heart in animal models (Giudice et al., 2000; Chu et al., 2002). There are some data that indicate that pathology that define the adult dystrophic phenotype originate in utero (Merrick et al., 2009). Here, we sought to determine if there is evidence of autonomic nervous system (ANS) abnormalities early in the life of $\delta$-sarcoglycan-deficient hamsters, a model of LGMD characterized by definitive histopathological and clinical sequelae of skeletal muscle pathology and heart failure late in life (Straub et al., 1998; Xiao et al., 2000; Zhu et al., 2005; Kato et al., 2006; Hoshijima et al., 2011).

\section{MATERIALS AND METHODS ANIMALS}

Male BIO F1B non-myopathic control and BIO TO-2 dystrophic hamsters were obtained from BioBreeders, Inc., Fitchburg, MA, USA. Handling and care of hamsters were consistent with federal guidelines and approved institutional protocols. Adults were studied at $\sim 9$ months, an age with established clinical and histopathological evidence of skeletal and cardiac muscle disease (Zhu et al., 
2005; Hoshijima et al., 2011). Newborn pups were studied at $\sim 4$ and $\sim 11$ days of age. The dams were removed from the nests prior to handling of the pups. A heat lamp was positioned near to a nest to provide ambient warmth to a pup in its mother's absence. The time required to position a pup on the heated ECG recording platform (LifeSpoon ${ }^{\mathrm{TM}}$, Mouse Specifics, Inc., Boston) and acquire the ECG signal was $\sim 5 \mathrm{~min}$, after which the pup was immediately returned to its dam.

\section{ECG RECORDINGS}

ECGs were recorded non-invasively in conscious hamsters similar to a method described previously for mice (Chu et al., 2001). Briefly, hamsters were gently removed from their cages and positioned inside the ECGenie ${ }^{\mathrm{TM}}$ recording enclosure (Mouse Specifics, Inc., Boston, MA, USA) sized to comfortably accommodate adult hamsters. A pair of silver-chloride ECG electrodes were embedded in the floor of the enclosure and spaced to provide contact between the electrodes and animals' paws. Since even modest handling of hamsters may induce alterations in heart rate, each hamster was permitted to acclimatize for $\sim 10 \mathrm{~min}$ prior to collection of data. The signals were digitized at a sampling rate of 2000 samples/s. When hamsters were positioned such that a forepaw and hind paw were not uniquely in contact with one of the electrodes, the output from the amplifier was discarded. Only data from continuous recordings were used in the analyses. Each signal was analyzed using e-MOUSE ${ }^{\mathrm{TM}}$, which incorporates Fourier analyses and linear time-invariant digital filtering of frequencies below $3 \mathrm{~Hz}$ and above $100 \mathrm{~Hz}$ to minimize environmental signal disturbances. The software uses a peak detection algorithm to find the peak of the $\mathrm{R}$-waves and to calculate heart rate. Subsequently, determination of first and second derivatives and algebraic "if-then" search the ECG signals for probable P-wave peaks and onset and termination of QRS complexes. The end of the T-wave of each signal was defined as the point where the signal returned to the isoelectric line (the mean voltage between the preceding P-wave and QRS interval). The QT intervals were rate corrected (QTc) by application of an equation recommended for rodent electrocardiography (Mitchell et al., 1998). The software plots its interpretation of P, $\mathrm{Q}, \mathrm{R}, \mathrm{S}$, and $\mathrm{T}$ for each beat so that spurious data resulting from unfiltered noise or motion artifacts may be rejected. e-MOUSE ${ }^{\mathrm{TM}}$ then calculates the mean of the ECG time intervals for each set of waveforms. Figure 1A depicts a F1B control hamster exploring an ECGenie $^{\mathrm{TM}}$ recording enclosure.

For electrocardiographic monitoring of the neonates, hamster pups were positioned atop of the LifeSpoon ${ }^{\mathrm{TM}}$ ECG recording module, the surface of which is embedded with two heated silverchloride electrodes to maintain a warmed environment similar to that provided by the nursing mother and nest. Surprisingly, even at $\sim 4$ days of age, the hamster pups were initially quite ambulatory, but became quiescent after $\sim 5 \mathrm{~min}$ at which time $\sim 20-30$ complexes were recorded.

Measures of heart beat-to-beat variability (HRV) were determined as the SD and the coefficient of variation $(\mathrm{CV})$. The SD reflects the dispersion about the average value for a parameter. $\mathrm{CV}$, expressed as a\%, was calculated from the following equation: $100 \times \mathrm{SD} /$ mean value. Power spectral analysis of ECG in the frequency domain for analysis of HRV was accomplished using low

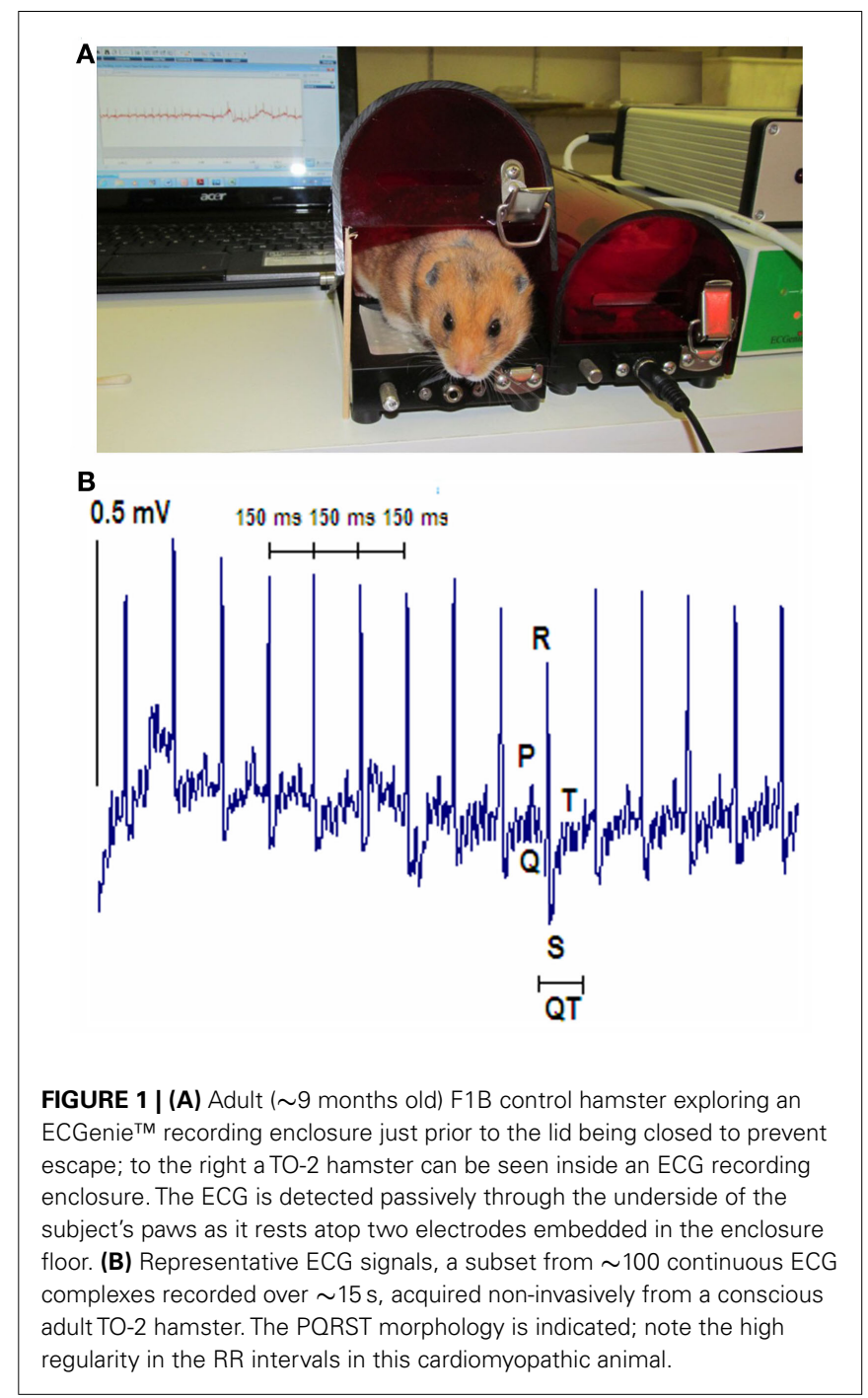

frequency (LF) and high frequency (HF) ranges similar to those previously published for hamsters (Giudice et al., 2000). The LF power range was set from 0.04 to $1.0 \mathrm{~Hz}$, and the $\mathrm{HF}$ power range was set from 1.0 to $3.0 \mathrm{~Hz}$.

\section{STATISTICS}

Data are presented as the mean \pm SE. Group means comparisons between strains, or among strains between ages, were performed using Student's two-tailed $t$-test for unpaired observations. Differences were considered significant with $P<0.05$.

\section{RESULTS}

We recorded ECGs non-invasively in 10 conscious F1B control hamsters and 10 TO-2 $\delta$-sarcoglycan-deficient hamsters, an animal model used routinely to test the efficacy of new therapies in treating muscular dystrophies and heart failure. A representative ECG recording from a $\sim 9$-month old TO-2 hamster is shown in Figure 1B, a subset of $\sim 100$ continuous ECG complexes acquired over $\sim 15 \mathrm{~s}$. Since the hamsters are conscious, baseline artifact and noise are apparent in the unfiltered signals. 
Yet, the P-waves and T-waves are discernible by eye and interpretable by the software algorithmic processing of the signal digitized at 2000 samples/s. Any discontinuities in the signal resulting from loss of contact between the paws and the electrodes were excluded from analysis. The hamster ECG is more typical of that of mouse and rat in that there is no separate $\mathrm{T}$ wave (Bestetti and Oliveira, 1990; Chu et al., 2001), and unlike in guinea pigs where there is a separate T-wave (Gussak et al., 2000).

Analyses of the digitized ECG signals demonstrated significantly higher heart rate in TO-2 dystrophic hamsters than in F1B controls ( $453 \pm 12$ bpm vs. $311 \pm 25$ bpm, $P<0.01$ ). Table 1 summarizes the ECG observations, which include shorter PR duration and QT interval in TO-2 than in F1B hamsters. Rate corrected QT (QTc) in TO-2 hamsters was significantly shorter than in F1B hamsters $(59.3 \pm 0.9 \mathrm{~ms}$ vs. $69.5 \pm 2.5 \mathrm{~ms}, P<0.05)$.

Heart rate variability was significantly lower in TO-2 hamsters compared to F1B control hamsters as reflected by HRV $(12.2 \pm 3.7 \mathrm{bpm}$ vs. $38.2 \pm 6.8 \mathrm{bpm}, P<0.05)$ and CV $(2.8 \pm 0.9 \%$ vs. $16.2 \pm 3.4 \%, P<0.05$; Table 1). Figure 2 illustrates $R R$ tachograms, or analysis of variations in the instantaneous heart rate time series using the beat-to-beat RR intervals, to reflect HRV. Note the lower beat-to-beat variability in the RR intervals in the cardiomyopathic TO-2 hamster, reflecting the lower HRV in these animals.

Analysis of HRV in the frequency domain further characterized reduced HRV in TO-2 hamsters. Fourier transformation of the beat-to-beat interval duration series yielded power spectral densities, from which total power, LF, and HF values were computed. Total power was reduced in cardiomyopathic TO-2 hamsters compared to controls $\left(3.0 \pm 1.2 \mathrm{~ms}^{2}\right.$ vs. $881.6 \pm 293.8 \mathrm{~ms}^{2}$, $P<0.05)$. The LF component, reflecting mainly sympathetic influences, was lower in TO-2 than in controls $\left(1.2 \pm 0.6 \mathrm{~ms}^{2}\right.$ vs. $\left.502.8 \pm 165.3 \mathrm{~ms}^{2}, P<0.05\right)$. The HF spectral component, reflecting mainly parasympathetic influences, was also lower in cardiomyopathic TO-2 than in F1B hamsters $\left(1.6 \pm 0.6 \mathrm{~ms}^{2}\right.$ vs. $\left.335.2 \pm 125.7 \mathrm{~ms}^{2}, P<0.05\right)$. Figure 3 illustrates the significantly lower HRV revealed by power spectral analysis in TO-2 dystrophic hamsters.

Table 1 | Electrocardiographic parameters in BIO F1B and BIOTO-2 conscious hamsters.

\begin{tabular}{lcc}
\hline & F1B $(\boldsymbol{n}=\mathbf{1 0})$ & TO-2 $(\boldsymbol{n}=\mathbf{1 0})$ \\
\hline Heart rate (bpm) & $311 \pm 25$ & $453 \pm 12^{*}$ \\
HR variability (bpm) & $38.2 \pm 6.8$ & $12.2 \pm 3.7^{*}$ \\
CV (\%) & $16.2 \pm 3.4$ & $2.8 \pm 0.9^{*}$ \\
PR (ms) & $53.8 \pm 4.2$ & $36.8 \pm 2.1^{*}$ \\
ORS (ms) & $23.5 \pm 1.7$ & $19.1 \pm 1.2$ \\
OT (ms) & $99.3 \pm 6.2$ & $68.5 \pm 1.9^{*}$ \\
OTc $(\mathrm{ms})$ & $69.5 \pm 2.5$ & $59.3 \pm 0.9^{*}$ \\
\hline
\end{tabular}

Values are mean $\pm S E$.

$H R$ var, heart rate variability; $C V$, coefficient of variation; OTC, QT corrected for heart rate.

${ }^{*} P<0.05$ vs. F1B hamsters.
Reducing the spacing of the electrodes and providing heat to the ECG recording surface enabled recording of the ECG in newborn F1B hamsters $(n=3)$ and TO-2 hamsters $(n=4)$ and return them to their dams. Figure $4 \mathrm{~A}$ depicts a hamster pup inside the LifeSpoon ${ }^{\mathrm{TM}}$ ECG recording chamber; Figure 4B shows representative complexes from a $\sim 4$-day old TO-2 hamster pup. The heart rate in the neonates, $208 \pm 11 \mathrm{bpm}$ in $\mathrm{F} 1 \mathrm{~B}$ pups and $248 \pm 15 \mathrm{bpm}$ in TO-2 pups, was significantly lower than heart rate in the adult hamsters $(P<0.05)$, consistent with the relative bradycardia that has been reported for neonatal mice (Heier et al., 2010) and rats (Hofer and Reiser, 1969). Heart rate was significantly higher, however, in $\sim 4$-day old TO-2 hamsters than F1B control hamsters $(P<0.05)$. HRV in F1B neonates was significantly lower than in adult F1B hamsters $(15.7 \pm 8.3$ bpm vs. $38.2 \pm 6.8, P<0.05)$. HRV
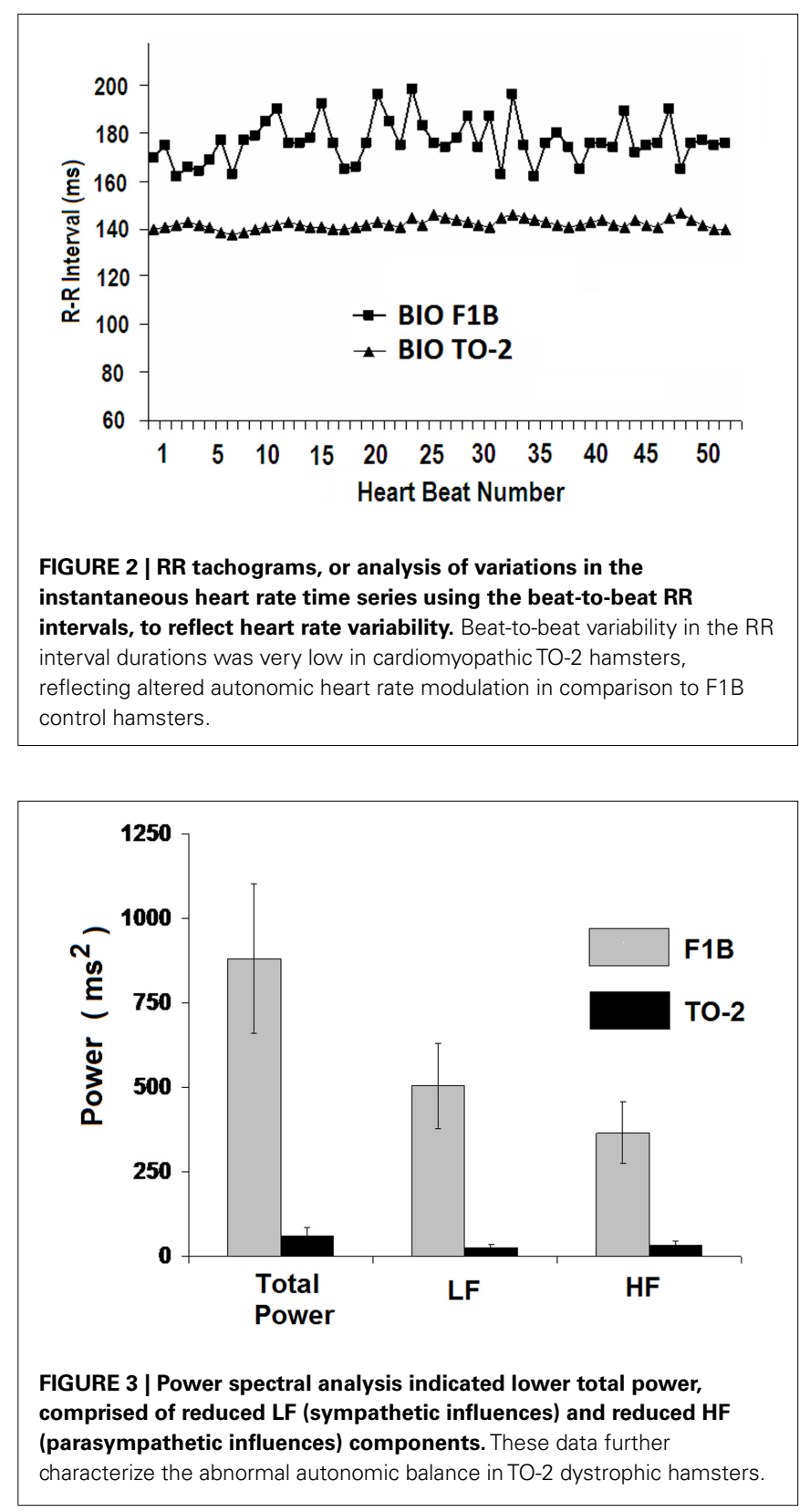


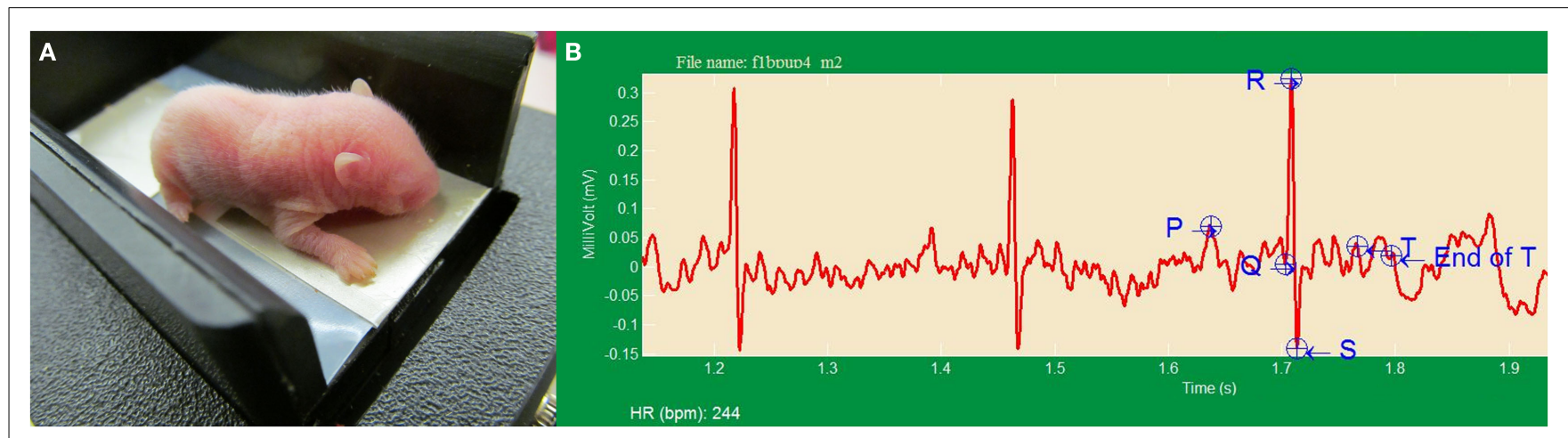

FIGURE 4 | (A) Neonate TO-2 hamster ( 4 days old) resting on warmed LifeSpoon ${ }^{\mathrm{TM}}$ ECG recording platform. (B) ECG tracings from neonatal ( 4 days old) F1B control hamster. Heart rate was significantly higher, and heart rate variability significantly lower, in TO-2 dystrophic hamsters, indicative of autonomic dysfunction, beginning very early in life. in developing TO-2 hamsters, moreover, was not different from adult TO-2 hamsters.

Autonomic differences were also apparent by $\sim 11$ days of age. HRV was significantly lower in TO-2 hamsters $(n=5)$ than in F1B hamsters $(n=5 ; 8.8 \pm 0.9 \mathrm{bpm}$ vs. $16.3 \pm 3.1 \mathrm{bpm}, P<0.05)$ as was $\mathrm{CV}(5.1 \pm 0.6 \%$ vs. $9.9 \pm 0.2 \%, P<0.05)$. Total power and the LF component were significantly lower in $~ 11$-day old TO-2 hamsters than in F1B controls. The ratio of the LF to HF component, a strong indicator of sympathetic tone (Mongue-Din et al., 2009), was also significantly lower in $\sim 11$-day old $\delta$-sarcoglycan-deficient TO-2 hamsters. These data indicate that autonomic dysfunction begins early in life in these overtly healthy animals that go on to develop pathological evidence and clinical signs of muscular dystrophy and heart failure.

\section{DISCUSSION}

This report describes a simple and elegant system for recording ECGs in conscious newborn and adult hamsters; neither attachment of wires, nor anesthesia, nor surgical implantation of devices is required. The ECG measurements we obtained non-invasively in conscious adult hamsters are comparable to those obtained using subcutaneously implanted electrodes (Giudice et al., 2000) and implantable telemetry devices (Mongue-Din et al., 2009; Wang et al., 2011). The data we obtained non-invasively in awake neonatal $\delta$-sarcoglycan-deficient TO-2 hamsters suggest that the ANS abnormalities clearly apparent in older moribund myopathic TO-2 animals may actually commence very early in life.

The $\delta$-sarcoglycan-deficient BIO TO-2 hamster is an established model of human LGMD-type 2F muscular dystrophy and heart failure. Whereas muscle weakness, predominantly affecting the pelvic and shoulder girdles, is the characteristic feature in patients with LGMD, cardiomyopathy is frequently the cause of death. The TO-2 hamster model recapitulates these characteristics. Pathology has indicated damage to the intercostal muscles, proximal limb muscles, muscles of the pelvic girdle, shoulder, gastrocnemius, triceps, and tibialis muscles (Straub et al., 1998; Xiao et al., 2000; Zhu et al., 2005). Skeletal muscle weakness and pathology are already evident in TO-2 animals at $\sim 1$ month of age (Homburger, 1979; Straub et al., 1998; Hampton et al., 2011). These hamsters, however, also develop cardiac pathology apparent after $\sim 3$ months of age. Cardiac fibrosis and calcification lead to dilation; congestive heart failure is usually the cause of death in TO-2 hamsters (Xiao et al., 2000; Zhu et al., 2005; Kato et al., 2006; Hoshijima et al., 2011).

Healthy modulation of the heart rhythm is influenced by the ANS, with contributions from the parasympathetic and sympathetic limbs of the ANS enabling a fair amount of plasticity in heart rate. Reduced HRV is an established marker of autonomic dysfunction and is associated with increased mortality in patients with heart failure. To the best of our knowledge, there are two descriptions of reduced HRV and autonomic dysfunction in TO-2 hamsters. Researchers (Giudice et al., 2000) described a procedure of surgical implantation of radio transmitters followed by a recovery period to report heart rate and HRV data quite similar to the values we obtained non-invasively. They demonstrated autonomic imbalance of cardiac control due to attenuation of parasympathetic activity and enhanced sympathetic tone in TO2 hamsters in heart failure. Our observations of reductions in the total spectral power, the LF component, and the HF component, are in agreement with those they reported. A subsequent study in cardiomyopathic TO-2 hamsters, also reporting similar values for heart rate and HRV as we report here, demonstrated that the lusitropic agent istaroxime improved cardiac function via preservation of autonomic tone (Lo Giudice et al., 2011). Changes in autonomic modulation of the heart in TO-2 cardiomyopathic hamsters was thought to be a reactive compensation to impaired cardiac function (Giudice et al., 2000). Our novel observations of autonomic dysregulation of heart rhythm in neonatal TO-2 hamsters, however, suggests that autonomic dysfunction might be a causative factor in the etiology of skeletal and cardiac muscle disease.

Autonomic nervous system dysfunction of the heart in TO-2 hamsters can be quickly and non-invasively quantified. To what extent unabated sympathetic overdrive commencing early in life contributes to the eventual demise of skeletal and cardiac muscle remains to be determined.

The method we describe for recording ECG in newborn rodents and our observations of neonatal autonomic disturbances are of 
practical significance in accelerating the testing of new therapeutic modalities in the treatment of muscular dystrophies and heart failure.

\section{REFERENCES}

Bestetti, R. B., and Oliveira, J. S. (1990). The surface electrocardiogram: a simple and reliable method for detecting overt and latent heart disease in rats. Braz. J. Med. Biol. Res. 23, 1213-1222.

Chu, V., Otero, J. M., Lopez, O., Morgan, J. P., Amende, I., and Hampton, T. G. (2001). Method for noninvasively recording electrocardiograms in conscious mice. BMC Physiol. 1, 6. doi:10.1186/1472-6793-1-6

Chu, V., Otero, J. M., Lopez, O., Sullivan, M. F., Morgan, J. P., Amende, I., and Hampton, T. G. (2002). Electrocardiographic findings in $\mathrm{mdx}$ mice: a cardiac phenotype of Duchenne muscular dystrophy. Muscle Nerve 26, 513-519.

Della Marca, G., Frusciante, R., Scatena, M., Testani, E., Vollono, C., Losurdo, A., Scarano, E., Colicchio, S., Farina, B., Gnoni, V., Mazza, S., Tonali, P. A., and Ricci, E. (2010). Heart rate variability in facioscapulohumeral muscular dystrophy. Funct. Neurol. 25, 211-216.

Giudice, P. L., Gagnol, J. P., Bellucci, A., Buffone, G., Careddu, A., Magni, G., Quagliata, T., Pacifici, L., and Carminati, P. (2000). Autonomic nervous system activity imbalance in cardiomyopathic hamster. J. Cardiovasc. Pharmacol. 36, 369-375.

Gussak, I., Chaitman, B. R., Kopecky, S. L., and Nerbonne, J. M. (2000). Rapid ventricular repolarization in rodents: electrocardiographic manifestations, molecular mechanisms, and clinical insights. J. Electrocardiol. 33, 159-170.

Hachiya, Y., Arai, H., Hayashi, M., Kumada, S., Furushima, W., Ohtsuka, E., Ito, Y., Uchiyama, A., and Kurata, K. (2005). Autonomic dysfunction in cases of spinal muscular atrophy type 1 with long survival. Brain Dev. 27, 574-578.

Hampton, T. G., Kale, A., Amende, I., Tang, W., McCue, S., Bhagavan, H. N., and VanDongen, C. G. (2011). Gait disturbances in dystrophic hamsters. J. Biomed. Biotechnol. 2011. doi:10.1155/2011/235354

Heier, C. R., Hampton, T. G., Wang, D., and Didonato, C. J. (2010).
Development of electrocardiogram intervals during growth of $\mathrm{FVB} / \mathrm{N}$ neonate mice. BMC Physiol. 10, 16. doi:10.1186/1472-6793-10-16

Hofer, M. A., and Reiser, M. F. (1969). The development of cardiac rate regulation in preweanling rats. Psychosom. Med. 31, 372-388.

Homburger, F. (1979). Myopathy of hamster dystrophy: history and morphologic aspects. Ann. N. Y. Acad. Sci. 317, 1-17.

Hoshijima, M., Hayashi, T., Jeon, Y. E., Fu, Z., Gu, Y., Dalton, N. D., Ellisman, M. H., Xiao, X., Powell, F. L., and Ross, J. Jr. (2011). Delta-sarcoglycan gene therapy halts progression of cardiac dysfunction, improves respiratory failure, and prolongs life in myopathic hamsters. Circ. Heart Fail. 4, 89-97.

Inoue, M., Mori, K., Hayabuchi, Y., Tatara, K., and Kagami, S. (2009). Autonomic function in patients with Duchenne muscular dystrophy. Pediatr. Int. 51, 33-40.

Judge, D. P., Kass, D. A., Thompson, W. R., and Wagner, K. R. (2011). Pathophysiology and therapy of cardiac dysfunction in Duchenne muscular dystrophy. Am. J. Cardiovasc. Drugs $11,287-294$.

Kato, Y., Iwase, M., Takagi, K., Nishizawa, T., Kanazawa, H., Matsushita, A., Umeda, H., Izawa, H., Noda, A., Koike, Y., Nagata, K., Yokota, M. (2006). Differential myolysis of myocardium and skeletal muscle in hamsters with dilated cardiomyopathy - beneficial protective effect of diltiazem. Circ. J. 70, 1497-1502.

Lo Giudice, P., Mattera, G. G., Gagnol, J. P., and Borsini, F. (2011). Chronic istaroxime improves cardiac function and heart rate variability in cardiomyopathic hamsters. Cardiovasc. Drugs Ther. 25, 133-138.

Marcello, N., Baratti, M., Ortaggio, F., Vescovini, E., Zanoni, P., Tugnoli, V., and De Grandis, D. (1995). Sympathetic skin response in patients with Duchenne muscular dystrophy. Electromyogr. Clin. Neurophysiol. 35, 387-395.

Margeta, M., Connolly, A. M., Winder, T. L., Pestronk, A., and Moore, S. A.

\section{ACKNOWLEDGMENTS}

Cecelia Hampton is acknowledged for her helpful comments on the manuscript.

(2009). Cardiac pathology exceeds skeletal muscle pathology in two cases of limb-girdle muscular dystrophy type 2I. Muscle Nerve 40, 883-889.

Merrick, D., Stadler, L. K., Larner, D. and Smith, J. (2009). Muscular dystrophy begins early in embryonic development deriving from stem cell loss and disrupted skeletal muscle formation. Dis. Model. Mech. 2 , 374-388.

Mitchell, G. F., Jeron, A., and Koren, G. (1998). Measurement of heart rate and Q-T interval in the conscious mouse. Am. J. Physiol. 274(Pt 2), H747-H751.

Mongue-Din, H., Salmon, A., Fiszman, M. Y., and Fromes, Y. (2009). Periodic variation in R-R intervals and cardiovascular autonomic regulation in young adult Syrian hamsters. Am. J. Physiol Regul. Integr. Comp. Physiol. 296, R610-R617.

Politano, L., Palladino, A., Nigro, G., Scutifero, M., and Cozza, V. (2008). Usefulness of heart rate variability as a predictor of sudden cardiac death in muscular dystrophies. Acta Myol. $27,114-122$.

Rakocević-Stojanović, V., Milovanović, B., Ivić, N., Ille, T., Marjanović, I., Stević, Z., Pavlović, S., and Lavrnić, D. (2007). Cardiac autonomic nervous system in patients with myotonic dystrophy type 1 . Acta Myol. 26, 112-114.

Rudnik-Schöneborn, S., Heller, R., Berg, C., Betzler, C., Grimm, T., Eggermann, T., Eggermann, K., Wirth, R., Wirth, B., and Zerres, K. (2008). Congenital heart disease is a feature of severe infantile spinal muscular atrophy. J. Med. Genet. 45, 635-638.

Straub, V., Duclos, F., Venzke, D. P. Lee, J. C., Cutshall, S., Leveille, C. J., and Campbell, K. P. (1998). Molecular pathogenesis of muscle degeneration in the $\delta$-sarcoglycandeficient hamster. Am. J. Pathol. 153, 1623-1630.

Wang, H., Siddharthan, V., Hall, J. O., and Morrey, J. D. (2011). Autonomic nervous dysfunction in hamsters infected with West Nile virus. PLoS
ONE 6, e19575. doi:10.1371/journal.pone.0019575

Xiao, X., Li, J., Tsao, Y. P., Dressman, D., Hoffman, E. P., and Watchko, J. F. (2000). Full functional rescue of a complete muscle (TA) in dystrophic hamsters by adeno-associated virus vector-directed gene therapy. J. Virol. 74, 1436-1442.

Yotsukura, M., Fujii, K., Katayama, A., Tomono, Y., Ando, H., Sakata, K., Ishihara, T., and Ishikawa, K. (1998). Nine-year follow-up study of heart rate variability in patients with Duchenne-type progressive muscular dystrophy. Am. Heart J. 136, 289-296.

Zhu, T., Zhou, L., Mori, S., Wang, Z., McTiernan, C. F., Qiao, C., Chen, C., Wang, D. W., Li, J., and Xiao, X. (2005). Sustained whole-body functional rescue in congestive heart failure and muscular dystrophy hamsters by systemic gene transfer. Circulation 112, 2650-2659.

Conflict of Interest Statement: T. G. Hampton is owner of Mouse Specifics, Inc., a company that has commercialized the non-invasive ECG instrumentation described in Materials and Methods. C. G. VanDongen is owner of BioBreeders, Inc., a purveyor of hamsters.

Received: 13 March 2012; accepted: 12 April 2012; published online: 18 May 2012.

Citation: Hampton TG, Kale A, McCue S, Bhagavan HN and VanDongen C (2012) Developmental changes in the ECG of a hamster model of muscular dystrophy and heart failure. Front. Pharmacol. 3:80. doi: 10.3389/fphar.2012.00080

This article was submitted to Frontiers in Pharmaceutical Medicine and Outcomes Research, a specialty of Frontiers in Pharmacology.

Copyright (c) 2012 Hampton, Kale, McCue, Bhagavan and VanDongen. This is an open-access article distributed under the terms of the Creative Commons Attribution Non Commercial License, which permits non-commercial use, distribution, and reproduction in other forums, provided the original authors and source are credited. 